\title{
FIXED POINT THEOREM AND SELF-SIMILARITY ON MIXED VICSEK PATTERNS
}

\author{
LEVENTE SIMON
}

Received 17 January, 2020

\begin{abstract}
The purpose if this paper is to present a fixed point result constructed by finite sequences. Using iterated function systems and related fractal operators, a mixed patterns generated by the a finite sequence patterns construct the sets of patterns built by black and white squares. A complete metric space related to a mixed pattern sequence is defined using the distance based on difference of the black squares' area.

The main result of the paper highlights that these fractal operators has unique fixed points for the sets generated by the mixed patterns. Moreover, the main theorem is also applied for Vicsek fractals such that results also hold for mixed Vicsek patterns. Motivated by various studies on growing graph sequences and related large structures, this paper underlines a new connection between fixed point theory and network science. Using circle patterns, the paper also interprets the main result on sets mixed patterns based on touching circles. Thus, the paper focuses a fixed point theorem on the sets mixed patterns built by iterated function systems and the distances calculated between the areas of these geometric shapes.
\end{abstract}

2010 Mathematics Subject Classification: 28A80; 37C25

Keywords: fixed point theorem, mixed patterns, interated function systems, Vicsek fractal, circle pattern

\section{INTRODUCTION}

Fractals are well known for describing growing processes in biology, physics and many other interdisciplinary topics too. Fixed point theorems [6] are strong and elegant tools for constructing self-similar sets and fractals, via the fractal operator theory and some iteration methods.

Growing mixed patterns [1-3] are self-similar geometrical sequences driven by a finite set of fixed patterns. An $n$-size pattern is constructed on the $n \times n$ sized grid with filled squares. Special cases of parameterized patterns are well known as the initial iteration of the Sierpiński carpet or the Vicsek fractal [8]. Thus, the growing pattern sequences based on these single patterns output widely known fractals.

Using iterated function systems and fractal operators, we interpret the limit of mixed pattern sequences using fixed point theory. We construct growing mixed sequences based on parameterized patterns built by squares. 
In operator theory, a bounded operator $T: X \rightarrow Y$ from the vector space $X$ to the vector space $Y$ is said to be a contraction if its norm is less or equal then 1 .

In complete metric spaces the Banach fixed point theorem guarantees the existence and uniqueness of fixed points for contraction operators. So, a map $T: X \rightarrow X$ is called a contraction mapping on the complete metric space $(X, d)$ if there exists $q \in[0,1)$ such that $d(T(x), T(y)) \leq q d(x, y)$.

Using the contraction principle we can construct fractals (or more precisely, prefractals), by an iterative procedure.

Mixed pattern can be constructed on other geometrical shapes too. As example, we confirm the results based on circle-driven patterns too. Moreover, specific growing mixed sequences are interpreted as mixed patterns based on the Vicsek fractal.

\section{MiXED PATTERNS AND ITERATED FUNCTION SYSTEMS}

Mixed patterns are constructed using simple patterns interpreted on unit squares. For any integer $m \geq 1$ let define $S_{i, j, m}=\left\{(x, y) \mid \frac{i}{m} \leq x \leq \frac{i+1}{m}\right.$ and $\left.\frac{j}{m} \leq y \leq \frac{j+1}{m}\right\}$ and $S_{m}=\left\{S_{i, j, k} \mid 0 \leq i \leq m-1\right.$ and $\left.0 \leq j \leq m-1\right\}$.

Let define the nonempty $A \in \mathcal{S}_{m}$ as an $m$-pattern with width $m$. Thus, an $m$-pattern is constructed by little squares $S_{i, j, m}$ corresponding with the ordered integer pair $(i, j)$ such that $A=S_{i_{1}, j_{1}, m} \cup S_{i_{2}, j_{2}, m} \cup \ldots$. Let also name the $S_{i, j, m}$ sets as black squares. Moreover, we also exclude the trivial case when $A$ is the whole unit square.

One way to construct mixed patters is with the help of iterated function systems. For any non-empty $A \in \mathcal{S}_{m}$ we define the mapping $f_{i, j, m}:[0,1]^{2} \rightarrow[0,1]^{2}$ as

$$
f_{i, j, m}(x, y)=\left(\frac{i}{m}, \frac{j}{m}\right)+\frac{1}{m}(x, y),
$$

for all $S_{i, j, m}$.
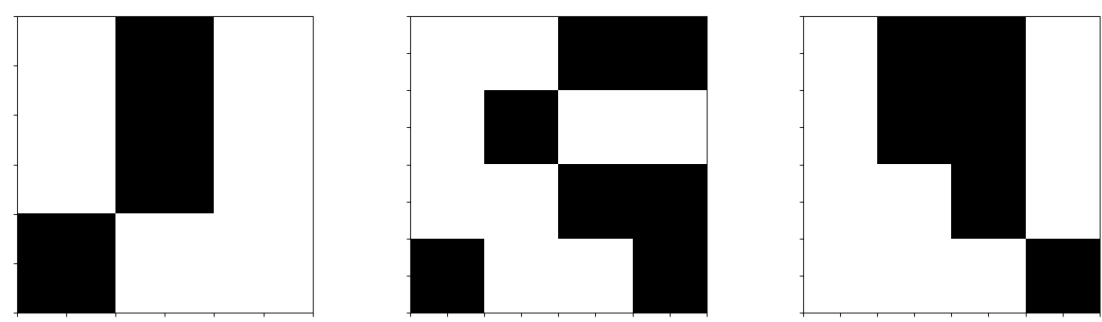

FIGURE 1. $m$-patterns: $\hat{f}_{A_{1}}\left([0,1]^{2}\right)$ (a 3-pattern), $\hat{f}_{A_{2}}\left([0,1]^{2}\right)$ and $\hat{f}_{A_{3}}\left([0,1]^{2}\right)$ (4-patterns). 
Definition 1. Let define the iterated function system $f_{A}=\left\{f_{i, j, m} \mid S_{i, j, m} \in A\right\}$ and the pattern-driven fractal operator $\hat{f}_{A}: P_{c p}\left([0,1]^{2}\right) \rightarrow P_{c p}\left([0,1]^{2}\right)$ as

$$
\hat{f}_{A}(Y)=\bigcup_{S_{i, j, m} \in A} f_{i, j, m}(Y), \quad \text { for all } \quad Y \in P_{c p}\left([0,1]^{2}\right)
$$

such that $Y$ is a compact square in the from the compact subsets of the unit square $P_{c p}\left([0,1]^{2}\right)$.

Let $\left\{A_{i}\right\}_{i=1}^{k}$ be a finite sequence of non-empty patterns with the width-sequence $\left\{m_{i}\right\}_{i=1}^{k} \cdot\left\{\hat{f}_{A_{i}}\right\}_{i=1}^{k}$ marks the corresponding iterated function system sequence. Let also use the notation for the multiplication $m(k)=m_{1} m_{2} \cdots m_{k}$.

For any fixed $A_{1}$ and $A_{2}$ the IFS $\hat{f}_{A_{1}}$ constructs the set $\hat{f}_{A_{1}}\left([0,1]^{2}\right) \subseteq[0,1]^{2}$. This pattern contains squares with side lengths equal with $\frac{1}{m_{1}}$ such that each mapping of the IFS generates a smaller black square from the unit square. Using the same construction, $\hat{f}_{A_{2}}\left(\hat{f}_{A_{1}}\left([0,1]^{2}\right)\right)$ is built by $m_{1} m_{2}$ little squares such that each element of the fractal operator $\hat{f}_{A_{2}}$ constructs squares with side length $\frac{1}{m_{1} m_{2}}$. Thus, $\left.\left(\hat{f}_{A_{2}} \circ \hat{f}_{A_{1}}\right)\left([0,1]^{2}\right)\right)$ generates a mixed pattern width value $m_{1} m_{2}$.

This implies that a sequence of $\left\{A_{i}\right\}_{i=1}^{k}$ patterns generates a mixed $m(k)$-pattern $\left(\hat{f}_{A_{k}} \circ \hat{f}_{A_{k-1}} \circ \cdots \circ \hat{f}_{A_{2}} \circ \hat{f}_{A_{1}}\right)$. Let us note the composed fractal operator as $\hat{F}_{k}$.

Definition 2. Let define the $m(k)$-pattern $M_{k}$ built by the different patterns $A_{1}, A_{2}, \ldots, A_{k}$ as a mixed pattern generated by a finite sequence of patterns.

Moreover, all of the patterns are defined on the same set $[0,1]^{2}$ and this brings the associativity on the composition of the corresponding fractal operator sequence $\left\{\hat{f}_{A_{i}}\right\}_{i=1}^{k}$.

In this paper we apply finite sequences of non-empty patterns on the set $[0,1]^{2}$ infinite times such that the end of a sequence is always followed by the first pattern. Thus, there exist the self-similar subsequence $[0,1]^{2}, \quad \hat{F}_{k}\left([0,1]^{2}\right)=$ $\left(\hat{f}_{A_{k}} \circ \hat{f}_{A_{k-1}} \circ \cdots \circ \hat{f}_{A_{2}} \circ \hat{f}_{A_{1}}\right)\left([0,1]^{2}\right), \hat{F}_{k}^{2}\left([0,1]^{2}\right)=\left(\hat{f}_{A_{k}} \circ \hat{f}_{A_{k-1}} \circ \cdots \circ \hat{f}_{A_{2}} \circ \hat{f}_{A_{1}}\right)^{2}\left([0,1]^{2}\right)$, ... constructed by the patterns.

\section{METRIC SPACES CONSTRUCTED BY MIXED PATTERNS}

We construct the set of mixed pattern $M_{k}$ based on the elements $\left\{A_{i}\right\}_{i=1}^{k}$. Using the iterated function systems let define the set as

$$
\begin{aligned}
\mathcal{F}_{k}=\left\{[0,1]^{2},\left(\hat{f}_{A_{k}} \circ \hat{f}_{A_{k-1}} \circ \cdots \circ \hat{f}_{1}\right)\left([0,1]^{2}\right)=\hat{F}_{k}\left([0,1]^{2}\right),\right. \\
\left.\left(\hat{f}_{A_{k}} \circ \hat{f}_{A_{k-1}} \circ \cdots \circ \hat{f}_{1}\right)^{2}\left([0,1]^{2}\right)=\hat{F}_{k}^{2}\left([0,1]^{2}\right), \hat{F}_{k}^{3}\left([0,1]^{2}\right), \ldots\right\} .
\end{aligned}
$$

The construction based on the set $[0,1]^{2}$ implies that all elements are built by little squares and it is easy to check that $\mathcal{A}_{k}$ is constructed as a unique sequence based on a set of mixed patterns. Thus, let us note the elements as the sequence

$$
\mathcal{F}_{k}=\left\{F_{0}=[0,1]^{2}, F_{1}=\hat{F}_{k}\left([0,1]^{2}\right), F_{2}, \ldots\right\} .
$$



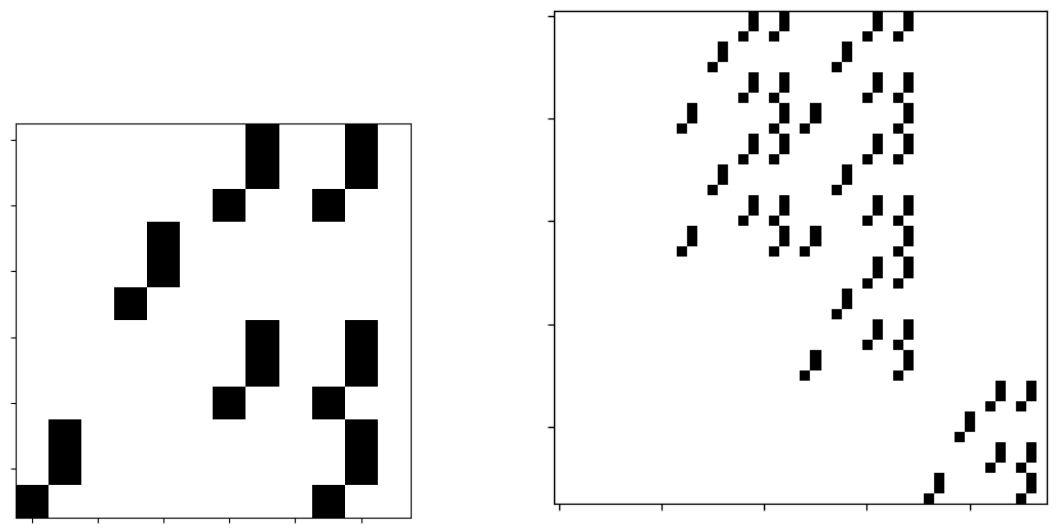

FIGURE 2. Mixed patters: $\hat{f}_{A_{2}}\left(\hat{f}_{A_{1}}\left([0,1]^{2}\right)\right)$ (a 12-pattern) and $\hat{f}_{A_{3}}\left(\hat{f}_{A_{2}}\left(\hat{f}_{A_{1}}\left([0,1]^{2}\right)\right)\right)$ (a 48-pattern).

Let us define the distance based the areas of the black squares.

Definition 3. Let note the construction of the element $F_{i}$ by using the black squares $f_{i}$. The distance between two elements is

$$
d\left(F_{u}, F_{v}\right)=\left|\sum_{f_{u} \in F_{u}}\left[\left[f_{u}\right]\right]-\sum_{f_{v} \in F_{v}}\left[\left[f_{v}\right]\right]\right|,
$$

where $k \in \mathbb{N}^{*}$ and indexes $u, v \in \mathbb{N}$. The operators $|\cdot|$ and $[[\cdot]]$ refer to absolute value and area of black squares, respectively.

We show that this distance constructs a metric space on the set $\mathcal{A}_{k}$.

Theorem 1. $\left(\mathcal{F}_{k}, d\right)$ is a metric space, where $\mathcal{A}_{k}=\left\{A_{i}\right\}_{i=1}^{k}, k \in \mathbb{N}^{*}$ is the set of mixed pattern and denotes the distance between the element of the pattern sequence generated by $\mathcal{A}_{k}$.

Proof. Let use the notation that $F_{i}$ is an $m(i)$-pattern built by the $n(i)$ number of squares. This means that $[[F(i)]]=n(i) \frac{1}{m(i)^{2}}$ such that $n(i) \leq m(i)^{2}$.

We calculate the difference between the areas of $F_{i}$ and $F_{i+1}$ for $i \in \mathbb{N}^{*}$ and $F_{i}, F_{i+1} \in \mathcal{F}_{k}$. Based on the construction, let be the $m_{i+1}$-pattern constructed by $n_{j+1}$ number of squares $A_{i+1}$ and the fractal operator $\hat{f}_{A_{i+1}}$ such that $F_{i+1}=\hat{f}_{A_{i+1}}\left(F_{i}\right), j=$ $1,2, \ldots, k$. This means that $\left[\left[F_{i+1}\right]\right]=\left(n(i) n_{i+1}\right) \frac{1}{m_{i}^{2} m_{i+1}^{2}}$. Based on the notation we get that $m(i+1)=m(i) m_{i+1}, n(i+1)=n(i) n_{i+1}$ and $\left[\left[F_{i+1}\right]\right]=n(i+1) \frac{1}{m(i+1)^{2}}$.

Thus 


$$
\begin{aligned}
d\left(F_{i}, F_{i+1}\right)=\mid n(i) \frac{1}{m(i)^{2}}-n(i) n_{i+1} & \frac{1}{\left(m(i) m_{i+1}\right)^{2}} \mid= \\
& =\left|\frac{n(i)}{m(i)^{2}}-\frac{n(i+1)}{m(i+1)^{2}}\right|=\frac{n(i)\left(m_{i+1}^{2}-n_{i+1}\right)}{m(i+1)^{2}},
\end{aligned}
$$

where $m_{i+1}^{2} \geq n_{i+1}$ and this implies that $\left[\left[F_{i}\right]\right] \geq\left[\left[F_{i+1}\right]\right]$ and $d\left(F_{i}, F_{i+1}\right)=$ $\frac{n(i)}{m(i)^{2}}-\frac{n(i+1)}{m(i+1)^{2}}$.

As the distance is defined, it is automatically verified that $d\left(F_{i}, F_{j}\right) \geq 0$ and $d\left(F_{i}, F_{j}\right)=d\left(F_{j}, F_{i}\right)$, for any $i, j \in \mathbb{N}, i \neq j$.

Last, but not least, let verify the triangle inequality such that $d\left(F_{i}, F_{j}\right) \geq$ $d\left(F_{i}, F_{k}\right)+d\left(F_{j}, F_{k}\right)$, where $i, j, k \in \mathbb{N}, i \neq j \neq k$ and $\left[\left[F_{i}\right]\right] \geq\left[\left[F_{i+1}\right]\right]$.

If $i \leq j \leq k$ then

$$
\begin{aligned}
d\left(F_{i}, F_{j}\right) & \leq d\left(F_{i}, F_{k}\right)+d\left(F_{j}, F_{k}\right) \\
\left|\frac{n(i)}{m(i)^{2}}-\frac{n(j)}{m(j)^{2}}\right| & \leq\left|\frac{n(i)}{m(i)^{2}}-\frac{n(k)}{m(k)^{2}}\right|+\left|\frac{n(j)}{m(j)^{2}}-\frac{n(k)}{m(k)^{2}}\right| \\
\frac{n(i)}{m(i)^{2}}-\frac{n(j)}{m(j)^{2}} & \leq \frac{n(i)}{m(i)^{2}}+\frac{n(j)}{m(j)^{2}}-2 \frac{n(k)}{m(k)^{2}} \\
\frac{n(i)}{m(i)^{2}}-\frac{n(j)}{m(j)^{2}} & \leq \frac{n(i)}{m(i)^{2}}+\frac{n(j)}{m(j)^{2}}-2 \frac{n(k)}{m(k)^{2}} \\
0 & \leq 2 \frac{n(j)}{m(j)^{2}}-2 \frac{n(k)}{m(k)^{2}} .
\end{aligned}
$$

Thus, we get the highlighted inequality $\left[\left[F_{j}\right]\right] \geq\left[\left[F_{k}\right]\right]$, where $j \leq k$.

Moreover, the case $i \leq k \leq j$ implies that

$$
\begin{aligned}
d\left(F_{i}, F_{j}\right) & \leq d\left(F_{i}, F_{k}\right)+d\left(F_{j}, F_{k}\right) \\
\frac{n(i)}{m(i)^{2}}-\frac{n(j)}{m(j)^{2}} & \leq \frac{n(i)}{m(i)^{2}}-\frac{n(k)}{m(k)^{2}}+\frac{n(k)}{m(k)^{2}}-\frac{n(j)}{m(j)^{2}}
\end{aligned}
$$

and this is equivalent with $d\left(F_{i}, F_{j}\right)=d\left(F_{i}, F_{j}\right)$. If $j \leq i \leq k$, then

$$
\begin{aligned}
d\left(F_{i}, F_{j}\right) & \leq d\left(F_{i}, F_{k}\right)+d\left(F_{j}, F_{k}\right) \\
\frac{n(j)}{m(j)^{2}}-\frac{n(i)}{m(i)^{2}} & \leq \frac{n(i)}{m(i)^{2}}-\frac{n(k)}{m(k)^{2}}+\frac{n(j)}{m(j)^{2}}-\frac{n(k)}{m(k)^{2}} \\
2 \frac{n(i)}{m(i)^{2}} & \geq 2 \frac{n(k)}{m(k)^{2}}
\end{aligned}
$$

equivalent with the showed inequality $\left[\left[F_{i}\right]\right] \geq\left[\left[F_{k}\right]\right]$ 
If $j \leq k \leq i$, then the inequality becomes equality

$$
\begin{aligned}
d\left(F_{i}, F_{j}\right) & \leq d\left(F_{i}, F_{k}\right)+d\left(F_{j}, F_{k}\right) \\
\frac{n(j)}{m(j)^{2}}-\frac{n(i)}{m(i)^{2}} & \leq \frac{n(k)}{m(k)^{2}}-\frac{n(i)}{m(i)^{2}}+\frac{n(j)}{m(j)^{2}}-\frac{n(k)}{m(k)^{2}}
\end{aligned}
$$

and this implies equality.

The case $k \leq i \leq j$ implies the inequality

$$
\begin{aligned}
d\left(F_{i}, F_{j}\right) & \leq d\left(F_{i}, F_{k}\right)+d\left(F_{j}, F_{k}\right) \\
\frac{n(i)}{m(i)^{2}}-\frac{n(j)}{m(j)^{2}} & \leq 2 \frac{n(k)}{m(k)^{2}}-\frac{n(j)}{m(j)^{2}}-\frac{n(i)}{m(i)^{2}} \\
2 \frac{n(i)}{m(i)^{2}} & \leq 2 \frac{n(k)}{m(k)^{2}}
\end{aligned}
$$

and the inequality $\left[\left[F_{k}\right]\right] \geq\left[\left[F_{j}\right]\right], k \leq i$ is checked.

If $k \leq j \leq i$ then the previous case can be followed as

$$
\begin{aligned}
d\left(F_{i}, F_{j}\right) & \leq d\left(F_{i}, F_{k}\right)+d\left(F_{j}, F_{k}\right) \\
\frac{n(j)}{m(j)^{2}}-\frac{n(i)}{m(i)^{2}} & \leq 2 \frac{n(k)}{m(k)^{2}}-\frac{n(j)}{m(j)^{2}}-\frac{n(i)}{m(i)^{2}} \\
2 \frac{n(j)}{m(j)^{2}} & \leq 2 \frac{n(k)}{m(k)^{2}}
\end{aligned}
$$

and we get the inequality $\left[\left[F_{k}\right]\right] \geq\left[\left[F_{i}\right]\right]$, where $k \leq i$.

Thus, the distance constructs the metric space $\left(\mathcal{F}_{k}, d\right)$.

\section{FIXED POINT RESULT}

A mixed pattern sequence $\left\{A_{k}\right\}$ and the distance $d$ construct the fractal operator $\hat{F}_{k}$. The operator has the fixed point in $\left(\mathcal{F}_{k}, d\right)$ if and only if there exists $F^{*} \in \mathcal{F}$ such that $\hat{F}_{k}\left(F^{*}\right)=F^{*}$.

As the lemma confirms, the metric space is complete.

Lemma 1. $\left(\mathcal{F}_{k}, d\right)$ is a complete metric space for any finite mixed pattern sequence $\left\{A_{k}\right\}$.

Proof. Based on Theorem 1, we need to show that arbitrary Cauchy sequence is convergent in the metric space $\left(\mathcal{F}_{k}, d\right)$. The construction implies that $\left[\left[\hat{f}_{A_{i}}(F)\right]\right] \leq[[F]]$ for every mixed pattern $F \in \mathcal{F}_{k}$ and for every pattern-driven fractal operator $\hat{f}_{A_{i}}$.

Based on the distance

$$
d\left(F_{u}, F_{v}\right)=\left|\sum_{f_{u} \in F_{u}}\left[\left[f_{u}\right]\right]-\sum_{f_{v} \in F_{v}}\left[\left[f_{v}\right]\right]\right|,
$$

where $F_{u}, F_{v} \in \mathcal{F}_{k}$ and these are built by the little squares $f_{i} \in F_{i}$ and $f_{j} \in F_{j}$, respectively. 
The construction implies one of the two cases: $F_{u}$ is an $m(u)$-pattern and $F_{v}$ is an $m(v)$-pattern such that $m(v)=m(u) m_{u+1} m_{u+2} \cdots m_{v}$ or $F_{v}$ is an $m(v)$-pattern and $F_{u}$ is an $m(v)$-pattern such that $m(u)=m(v) m_{v+1} m_{v+2} \cdots m_{u}$.

As the metric symmetry implies, we need to check only the first case. If $u<v, F_{u}$ is an $m(u)$-pattern, $F_{v}$ is an $m(v)$-pattern and $m(v)=m(u) m_{u+1} m_{u+2} \cdots m_{v}$ then

$$
d\left(F_{u}, F_{v}\right)=\frac{n_{1} n_{2} \cdots n_{u}}{m_{1}^{2} m_{2}^{2} \cdots m_{u}^{2}}-\frac{n_{1} n_{2} \cdots n_{u} n_{u+1} \cdots n_{v}}{m_{1}^{2} m_{2}^{2} \cdots m_{u}^{2} m_{u+1}^{2} \cdots m_{v}},
$$

where $n_{i}<m_{i}^{2}, i \in\{1,2, \ldots, u, u+1, \ldots, v\}$. Thus, for all $\varepsilon>0$ there exists $N=N(\varepsilon) \in \mathbb{N}^{*}$ such that $\left[\left[F_{N}\right]\right]=\frac{n_{1} n_{2} \ldots n_{N}}{m_{1}^{2} m_{2}^{2} \ldots m_{N}^{2}}<\varepsilon$. This implies, that for all $N(\varepsilon) \leq u \leq v$ hold the inequality $0 \leq\left[\left[F_{v}\right]\right] \leq\left[\left[F_{u}\right]\right] \leq \varepsilon$ and

$$
d\left(F_{u}, F_{v}\right) \leq\left[\left[F_{u}\right]\right]-\left[\left[F_{v}\right]\right] \leq\left[\left[F_{u}\right]\right]<\varepsilon .
$$

This and the symmetry implies that arbitrary Cauchy sequence is convergent in the metric space $\left(\mathcal{F}_{k}, d\right)$ and the metric space is complete.

Let consider the metric space $\left(\mathcal{F}_{k}, d\right)$ and the fractal operator $\hat{F}_{k}$ constructed by a sequence of patterns $\left\{A_{1}, A_{2}, \ldots, A_{k}\right\}$.

As it is defined, the space $\mathcal{F}_{k}$ is build such that the fractal operator transforms the element $F_{i}$ to $\hat{F}_{k}\left(F_{i}\right)=F_{i+1}$, where $F_{i}$ is an $m(i)$-pattern, $F_{i+1}$ is an $m(i+k)$-pattern, $m(i+k)=m(i) m_{1} m_{2} \cdots m_{k}$ and $i \in \mathbb{N}$.

The contraction principle implies the following theorem.

Theorem 2. The pattern-driven fractal operator $\hat{F}_{k}$ has an unique fixed point on the metric space $\left(\mathcal{F}_{k}, d\right)$.

Proof. As the composition implies $\hat{F}_{k}(F)=\frac{n_{1}}{m_{1}^{2}} \frac{n_{2}}{m_{2}^{2}} \cdots \frac{n_{k}}{m_{k}^{2}}[[F]]$ for all $S \in \mathcal{F}_{k}$.

Let consider the elements $F_{i}$, and $F_{j}$ from the complete metric space $\mathcal{F}_{k}$ such that $i \leq j$ and $i, j \in \mathbb{N}$. Using Theorem 1 , we get that

$$
\begin{aligned}
d\left(\hat{F}_{k}\left(F_{i}\right), \hat{F}_{k}\left(F_{j}\right)\right)=\left|\frac{n_{1} n_{2} \cdots n_{k}}{m_{1}^{2} m_{2}^{2} \cdots m_{k}^{2}}\left[\left[F_{i}\right]\right]-\frac{n_{1} n_{2} \cdots n_{k}}{m_{1}^{2} m_{2}^{2} \cdots m_{k}^{2}}\left[\left[F_{j}\right]\right]\right| & = \\
& =\left|\frac{n_{1} n_{2} \cdots n_{k}}{m_{1}^{2} m_{2}^{2} \cdots m_{k}^{2}}\right| d\left(F_{i}, F_{j}\right),
\end{aligned}
$$

where $i, j \in \mathbb{N}$ and $\frac{n_{1} n_{2} \ldots n_{k}}{m_{1}^{2} m_{2}^{2} \ldots m_{k}^{2}}<1$ is Lipschitz parameter with the conditions $n_{i+1}<m_{i+1}^{2}$ and $n_{j+1}<m_{j+1}^{2}$.

Thus, fractal operator $\hat{F}_{k}$ is a Lipschitz-function on $\left(\mathcal{F}_{k}, d\right)$ and the contraction principle implies that it has an unique fixed point. Furthermore, the fixed point $F^{*}$ can be found as follows: start with an arbitrary element $F_{i} \in \mathcal{F}_{k}$ and use the sequence defined as $F_{i}, \hat{F}_{k}\left(F_{i}\right)=F_{i+1}, \hat{F}_{k}\left(F_{i+1}\right)=F_{i+2}, \ldots$, then $i \rightarrow+\infty$ implies that $F_{i} \rightarrow F^{*}$. 
As a conclusion, we highlighted the fractal operator $\hat{F}_{k}$ generated by the pattern sequence has an unique fixed point.

\section{MiXed CIRCLE PATTERNS}

Mixed circle patterns start from the compact unit circle $\operatorname{circ}(0,1)$ and an example can be constructed using three patterns: the pattern $C_{1}$ constructs with 5 little circles, $C_{2}$ operates with 9 and $C_{3}$ works with 13 .

Thus, let also define the functions

$$
\begin{gathered}
f_{C_{1}}=\left\{f_{1}(x, y)=\frac{1}{5}(x, y), f_{2}(x, y)=\left(0, \frac{2}{3}\right)+\frac{1}{3}(x, y),\right. \\
f_{3}(x, y)=\left(0,-\frac{2}{3}\right)+\frac{1}{3}(x, y), f_{4}(x, y)=\left(\frac{2}{3}, 0\right)+\frac{1}{3}(x, y), \\
\left.f_{5}(x, y)=\left(-\frac{2}{3}, 0\right)+\frac{1}{3}(x, y)\right\},
\end{gathered}
$$

the iterated function system $f_{C_{1}}=\left\{f_{1}, f_{2}, f_{3}, f_{4}, f_{5}\right\}$ and the corresponding fractal operator

$$
\hat{f}_{C_{1}}(Y)=f_{1}(Y) \cup f_{2}(Y) \cup f_{3}(Y) \cup f_{4}(Y) \cup f_{5}(Y), \quad \text { for all } \quad Y \in P_{c p}(\operatorname{circ}(0,1)) .
$$

On the other hand, the circle pattern $C_{2}$ and $C_{3}$ are based on the mappings

$$
\begin{aligned}
& f_{C_{2}}=\left\{f_{1}(x, y)=\frac{1}{5}(x, y), f_{2}(x, y)=\left(0, \frac{2}{5}\right)+\frac{1}{5}(x, y),\right. \\
& f_{3}(x, y)=\left(0,-\frac{2}{5}\right)+\frac{1}{5}(x, y), f_{4}(x, y)=\left(\frac{2}{5}, 0\right)+\frac{1}{5}(x, y), \\
& f_{5}(x, y)=\left(-\frac{1}{5}, 0\right)+\frac{1}{5}(x, y), f_{6}(x, y)=\left(0, \frac{2}{5}\right)+\frac{1}{5}(x, y), \\
& f_{7}(x, y)=\left(0,-\frac{2}{5}\right)+\frac{1}{5}(x, y), f_{8}(x, y)=\left(-\frac{1}{5}, 0\right)+\frac{1}{5}(x, y), \\
&\left.f_{9}(x, y)=\left(-\frac{2}{5}, 0\right)+\frac{1}{5}(x, y)\right\} .
\end{aligned}
$$

These construct the iterated function system $f_{C_{2}}=\left\{f_{1}, f_{2}, \ldots, f_{13}\right\}$ and the corresponding fractal operator

$$
\hat{f}_{C_{2}}(Y)=f_{1}(Y) \cup f_{2}(Y) \cup \cdots \cup f_{13}(Y), \quad \text { for all } \quad Y \in P_{c p}(\operatorname{circ}(0,1)) .
$$

and

$$
\begin{aligned}
f_{C_{3}}=\left\{f_{1}(x, y)=\frac{1}{5}(x, y), f_{2}(x, y)=\left(0, \frac{2}{5}\right)+\frac{1}{5}(x, y),\right. \\
f_{3}(x, y)=\left(0,-\frac{2}{5}\right)+\frac{1}{5}(x, y), f_{4}(x, y)=\left(\frac{2}{5}, 0\right)+\frac{1}{5}(x, y), \\
f_{5}(x, y)=\left(-\frac{1}{5}, 0\right)+\frac{1}{5}(x, y), f_{6}(x, y)=\left(0, \frac{2}{5}\right)+\frac{1}{5}(x, y),
\end{aligned}
$$




$$
\begin{array}{r}
f_{7}(x, y)=\left(0,-\frac{2}{5}\right)+\frac{1}{5}(x, y), f_{8}(x, y)=\left(\frac{2}{5}, 0\right)+\frac{1}{5}(x, y), \\
f_{9}(x, y)=\left(-\frac{2}{5}, 0\right)+\frac{1}{5}(x, y), f_{10}(x, y)=\left(-\frac{1}{5}, \frac{1}{5}\right)+\frac{1}{5}(x, y) \\
f_{11}(x, y)=\left(\frac{1}{5}, \frac{1}{5}\right)+\frac{1}{5}(x, y), f_{12}(x, y)=\left(\frac{1}{5},-\frac{1}{5}\right)+\frac{1}{5}(x, y), \\
\left.f_{13}(x, y)=\left(-\frac{1}{5},-\frac{1}{5}\right)+\frac{1}{5}(x, y)\right\} .
\end{array}
$$

These construct the iterated function system $f_{C_{2}}=\left\{f_{1}, f_{2}, \ldots, f_{13}\right\}$ and the corresponding fractal operator

$$
\hat{f}_{C_{2}}(Y)=f_{1}(Y) \cup f_{2}(Y) \cup \cdots \cup f_{13}(Y), \quad \text { for all } \quad Y \in P_{c p}(\operatorname{circ}(0,1)) .
$$

Based on the multipliers, we interpret $C_{1}$ as a 3-pattern, $C_{2}$ and $C_{3}$ as a 5-pattern. We base the mixed circle pattern $C$ on the elements $C_{1}, C_{2}$ and $C_{3}$ such that

$$
\begin{aligned}
C=\{\operatorname{circ}(0,1), & \left(\hat{C}_{3} \circ \hat{C}_{2} \circ \hat{C}_{1}\right)(\operatorname{circ}(0,1))=\hat{C}\left([0,1]^{2}\right), \\
\left(\hat{C}_{3} \circ \hat{C}_{2} \circ \hat{C}_{1}\right)^{2}(\operatorname{circ}(0,1)) & \left.=\hat{C}^{2}(\operatorname{circ}(0,1)), \hat{C}^{3}(\operatorname{circ}(0,1)), \ldots\right\}
\end{aligned}
$$

Thus, the operators $\hat{C}_{1}, \hat{C}_{2}$ and $\hat{C}_{3}$ form the fractal operator $\hat{C}$. The construction based on the unit square $[0,1]^{2}$ implies also for this case that the mixed circle pattern constructs the sequence

$$
\mathcal{C}=\left\{C_{0}=\operatorname{circ}(0,1), C_{1}=\hat{C}(\operatorname{circ}(0,1)), C_{2}=\hat{C}^{2}(\operatorname{circ}(0,1)), \ldots\right\} .
$$
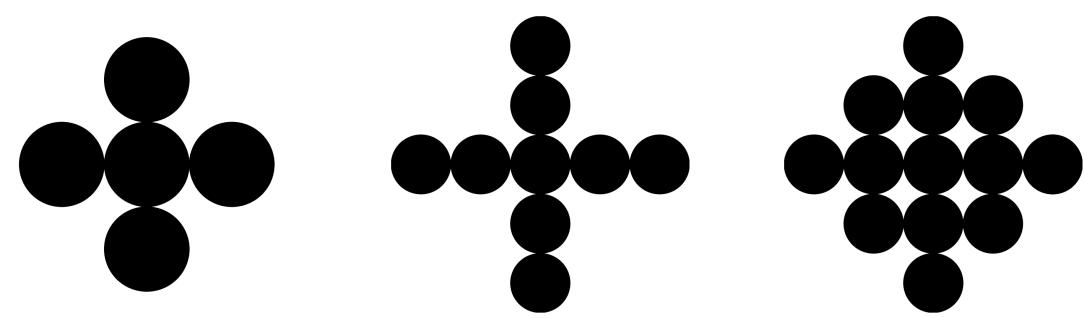

FIGURE 3. $m$-patterns: $\hat{f}_{C_{1}}(\operatorname{circ}(0,1))$ (3-pattern), $\hat{f}_{C_{2}}(\operatorname{circ}(0,1))$ (5-pattern) and $\hat{f}_{C_{3}}(\operatorname{circ}(0,1))$ (5-pattern). 

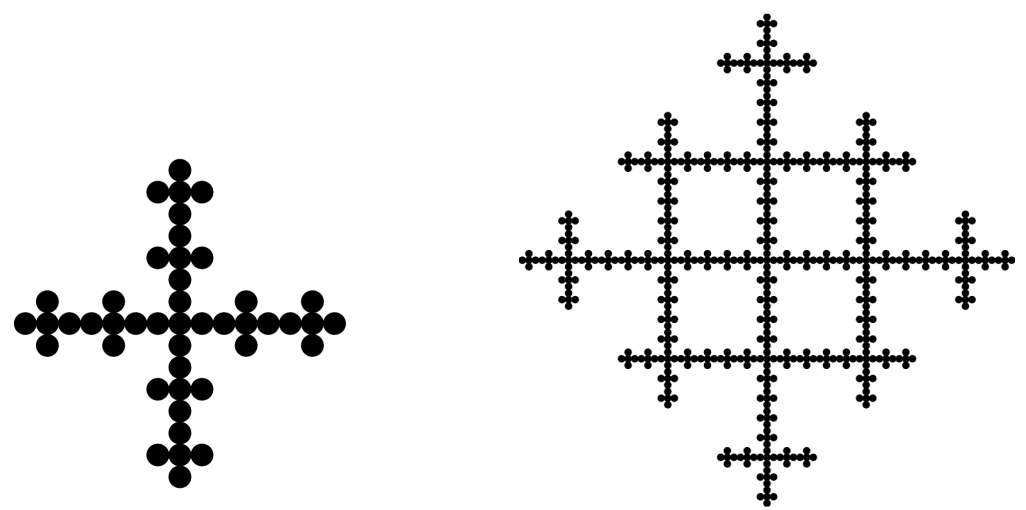

FIGURE 4. Mixed patters: $\hat{f}_{C_{2}}\left(\hat{f}_{C_{1}}(\operatorname{circ}(0,1))\right)$ (a 15-pattern) and $\hat{f}_{C_{3}}\left(\hat{f}_{C_{2}}\left(\hat{f}_{C_{1}}(\operatorname{circ}(0,1))\right)\right)$ (a 75-pattern).

Let us define the distance based on the areas of circles.

Definition 4. Let note the construction of the element $C_{i}$ by using the black circles $c_{i}$. The distance between two elements is

$$
d_{C}\left(C_{u}, C_{v}\right)=\left|\sum_{c_{u} \in C_{u}}\left[\left[c_{u}\right]\right]-\sum_{c_{v} \in C_{v}}\left[\left[c_{v}\right]\right]\right|,
$$

where $k \in \mathbb{N}^{*}$ and the indexes $u, v \in \mathbb{N}$. The operators $|\cdot|$ and $[[\cdot]]$ refer to absolute value and area, respectively.

Our results imply that this distance builds a metric space on $C$.

Theorem 3. $\left(C, d_{C}\right)$ is a metric space, where $d_{C}$ denotes the distance between two elements of the pattern sequence generated by $C$.

Moreover, we also confirm that the metric space is complete.

Lemma 2. $\left(C, d_{C}\right)$ is a complete metric space for any finite mixed pattern sequence $\left\{C_{k}\right\} \subseteq C$.

The metric space $\left(C, d_{C}\right)$ and the fractal operator $\hat{C}$ constructs the set $\mathcal{C}$ such that the fractal operator transforms the element $C_{i}$ to $\hat{C}\left(C_{i}\right)=C_{i+1}$. Using the contraction principle and Theorem 2 we obtain the following theorem. C.

Theorem 4. The fractal operator $\hat{C}$ has an unique fixed point on the metric space

Thus, the fractal operator $\hat{C}$ extends the results for circle patterns. 

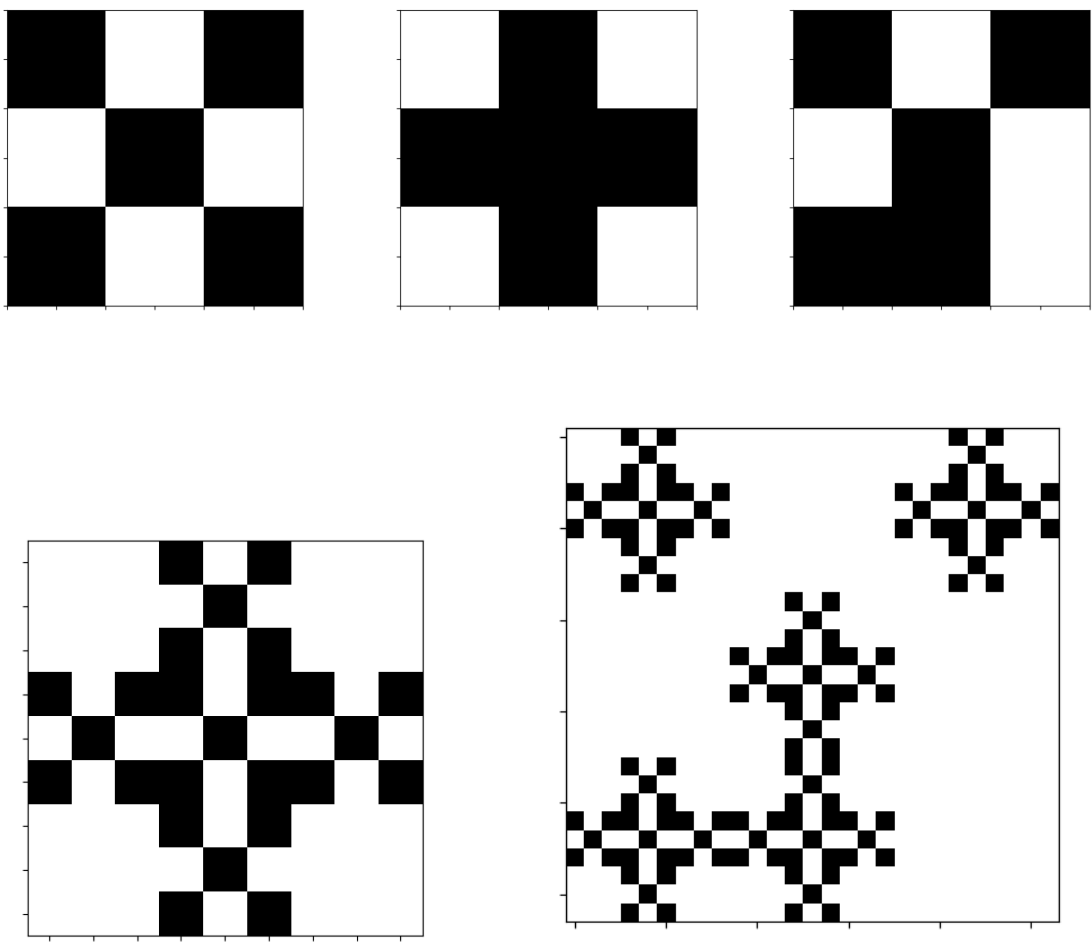

FIGURE 5. Vicsek patters: $\hat{f}_{V_{1}}\left([0,1]^{2}\right), \hat{f}_{V_{2}}\left([0,1]^{2}\right), \hat{f}_{V_{3}}\left([0,1]^{2}\right)$, $\hat{f}_{V_{2}}\left(\hat{f}_{V_{1}}\left([0,1]^{2}\right)\right)$ (a 9-pattern) and $\hat{f}_{V_{3}}\left(\hat{f}_{V_{2}}\left(\hat{f}_{V_{1}}\left([0,1]^{2}\right)\right)\right.$ ) (a 27pattern).

\section{INTERPRETATION ON VICSEK PATTERNS}

Studies on Vicsek fractals [7, 8] and related growing patterns mainly focus on spectral [4,9] or network coherence [5]. We highlight a fixed point result based on a finite sequence of Vicsek fractals.

The Vicsek fractal arise from a construction based on the Sierpinski carpet. It started from the 3-pattern $V_{1}=\left\{S_{0,0,3}, S_{0,2,3}, S_{1,1,3}, S_{2,0,3}, S_{2,2,3}\right\}$. Its cross form $V_{2}=\left\{S_{0,1,3}, S_{1,0,3}, S_{1,1,3}, S_{1,2,3}, S_{1,1,3}\right\}$ is also named as antenna fractal.

Let us define the sequence of Vicsek fractals $\mathcal{V}_{k}=\left\{V_{1}, V_{2}, \ldots, V_{k}\right\}$ and the corresponding operators $\hat{f}_{V_{1}}, \hat{f}_{V_{2}}, \ldots, \hat{f}_{V_{k}}$. The sequence generates a mixed Vicsek pattern and the corresponding fractal operator $\hat{F}_{V_{k}}$. We also define the set $\mathcal{V}_{k}$ generated by the fractal operated of the mixed pattern.

By the fixed point theorem 2, we also get the following corollary for mixed Vicsek patterns. 
Corollary 1. The fractal operator $\hat{F}_{V_{k}}$ based on the mixed pattern constructed by a finite sequence of Vicsek fractals has an unique fixed point on the metric space $\left(\mathcal{V}_{\|}, d\right)$

Moreover, let us change elements of the Vicsek pattern with circles. It is easy to check that we can interpret the corollary with circles too. Thus, we define the set of Vicsek 3-size circle patterns $\mathcal{C}_{k}=\hat{C}_{1}, \hat{C}_{2}, \ldots, \hat{C}_{k}$ and the fractal operator $\hat{F}_{C_{k}}$. This implies the corollary corresponding with the circle based construction.

Corollary 2. The fractal operator $\hat{F}_{C_{k}}$ based on the mixed pattern constructed by a finite sequence of Vicsek circle fractals has an unique fixed point on the metric space $\left(C_{k}, d_{C}\right)$

Thus, there is gotten a fixed point for the fractal operator corresponding with the mixed Vicsek patterns and mixes Vicsek circle patterns too.

\section{CONCLUSION}

Our research highlighted fixed point theorems on mixed patterns. Using iterated function systems, we built growing sequences based on square size grids and we extended the results for circle-driven patterns too. We also constructed complete metric spaces on the sets driven by the mixed patters.

As underlined result, we showed that the fractal operators corresponding with the iterated function systems have unique fixed point on the sets build by the mixed patterns.

We spotlighted the result on mixed pattern driven by Vicsek fractals too such that we showed that specific mixed patters may be interpreted as mixed Vicsek patterns.

\section{REFERENCES}

[1] L.-L. Cristea and G. Leobacher, "On the length of arcs in labyrinth fractals," Monatshefte für Mathematik, vol. 185, no. 4, pp. 575-590, 2018, doi: 10.1007/s00605-017-1056-8. [Online]. Available: https://doi.org/10.1007/s00605-017-1056-8

[2] L.-L. Cristea and B. Steinsky, "Curves of infinite length in 4x4-labyrinth fractals," Geometriae Dedicata, vol. 141, no. 1, pp. 1-17, 2009, doi: 10.1007/s10711-008-9340-3. [Online]. Available: https://doi.org/10.1007/s10711-008-9340-3

[3] L.-L. Cristea and B. Steinsky, "Curves of infinite length in labyrinth fractals," Proceedings of the Edinburgh Mathematical Society, vol. 54, no. 02, pp. 329-344, 2011, doi: 10.1017/S0013091509000169.

[4] Y. Hu, D.-C. Tian, and J.-Q. You, "Spectral properties of the vicsek fractal," Phys. Rev. $B$, vol. 53, pp. 5070-5073, 1996, doi: 10.1103/PhysRevB.53.5070. [Online]. Available: https://link.aps.org/doi/10.1103/PhysRevB.53.5070

[5] S. Patterson and B. Bamieh, "Network coherence in fractal graphs," in Proceedings of the IEEE Conference on Decision and Control," doi: 10.1109/CDC.2011.6161307, 2011, pp. 6445-6450. [Online]. Available: https://doi.org/10.1109/CDC.2011.6161307

[6] I. A. Rus, A. Petruşel, and G. Petruşel, Fixed Point Theory. Cluj-Napoca: Cluj University Press, 2008. 
[7] T. Vicsek, "Fractal models for diffusion controlled aggregation," J. Phys. A Math. Gen., vol. 16, no. 17, pp. 647-652, 1983, doi: 10.1088/0305-4470/16/17/003. [Online]. Available: https://doi.org/10.1088\%2F0305-4470\%2F16\%2F17\%2F003

[8] T. Vicsek, Fractal Growth Phenomena. $\quad$ Singapore - New Jersey: World Scientific, $1989 . \quad$ doi: 10.1007/978-1-4614-6946-9.

[9] W. Xiaomint, L. Zhifangt, S. Suenchingt, and R. Taot, "Studies of the spectral dimension of the Vicsek snowflake fractal," J. Phys. A Math. Gen., vol. 24, no. 23, pp. 1353-1357, 1991, doi: 10.1088/0305-4470/24/23/006. [Online]. Available: https://iopscience.iop.org/article/10.1088/ 0305-4470/24/23/006/meta

\section{Author's address}

\section{Levente Simon}

Babeş-Bolyai University, Faculty of Mathematics and Computer Science, 1 M. Kogălniceanu St., 400084 Cluj-Napoca, Romania

E-mail address: simonlemath.ubbcluj.ro 